\title{
Solving generalized eigenvalue problems on the interfaces to build a robust two level FETI method
}

Une grille grossière robuste pour FETI grâce à la résolution de problèmes aux valeurs propres généralisés sur les interfaces.

\author{
Nicole Spillane ${ }^{\mathrm{a}, \mathrm{b}}$ Victorita Dolean ${ }^{\mathrm{c}}$ Patrice Hauret ${ }^{\mathrm{b}}$ Frédéric Nataf $^{\mathrm{a}}$, Daniel Rixen ${ }^{\mathrm{d}}$ \\ ${ }^{\mathrm{a}}$ Laboratoire Jacques Louis Lions, UMR 7598, UPMC Université Paris 6, F-75252 Paris, France \\ ${ }^{\mathrm{b}}$ Michelin Technology Center, Place des Carmes-Déchaux, 63000 Clermont-Ferrand, France \\ ${ }^{\mathrm{c}}$ Laboratoire J.-A. Dieudonné, UMR 6621, Université de Nice-Sophia Antipolis, 06108 Nice Cedex 02, France \\ ${ }^{\mathrm{d}}$ Institute of Applied Mechanics, TU München, D-85747 Garching, Germany \\ Received ${ }^{* * * * *}$; accepted after revision +++++ \\ Presented by
}

\begin{abstract}
FETI is a very popular method which has proved to be extremely efficient on many large scale industrial problems. One drawback is that it performs best when the decomposition of the global problem is closely related to the parameters in equations. This is somewhat confirmed by the fact that the theoretical analysis goes through only if some assumptions on the coefficients are satisfied. We propose here to build a coarse space for which the convergence rate of the two level method is guaranteed regardless of any additional assumptions. We do this by identifying the problematic modes using generalized eigenvalue problems.
\end{abstract}

To cite this article: , C. R. Acad. Sci. Paris, Ser. I $+++++(+++++)$.

\section{Résumé}

La méthode FETI a demontré son efficacité et sa compétitivité sur de nombreux problèmes industriels. Un désavantage est que ses performances dépendent fortement de la distribution des coefficients dans les équations. Ceci est en quelque sorte confirmé par le fait que l'analyse théorique requiert des hypothèses sur ces coefficients et le partitionnement. Nous proposons ici la construction d'un espace grossier telle que le taux de convergence de la méthode à deux niveaux soit garanti sans hypothèses supplémentaire. Cette construction repose sur l'identification des modes problématiques grâce à la résolution de problèmes aux valeurs propres généralisés.

Pour citer cet article : , C. R. Acad. Sci. Paris, Ser. I $+++++(+++++)$.

\footnotetext{
Email addresses: spillane@ann.jussieu.fr (Nicole Spillane), dolean@unice.fr (Victorita Dolean), patrice.hauret@fr.michelin.com (Patrice Hauret), nataf@ann.jussieu.fr (Frédéric Nataf), rixen@tum.de (Daniel Rixen).
} 
Version française abrégée

Il est assez intuitif de comprendre que rajouter des vecteurs dans un espace grossier améliore la convergence de la méthode à deux niveaux correspondante puisque dans le cas extrême où l'espace grossier contient l'espace initial complet la résolution devient directe. Il est plus difficile d'identifier une grille grossière qui offre un bon compromis entre sa taille et l'efficacité de la méthode qu'elle engendre. Dès [7] l'idée d'utiliser des problèmes aux valeurs propres (non généralisés) émerge pour obtenir un taux de convergence choisi a priori. Cependant la pratique montre que l'espace grossier obtenu est très grand. Dans le cas de l'équation (scalaire) de Darcy [2] propose de résoudre des problèmes aux valeurs propres généralisés pour identifier les modes qui ralentissent la convergence. Ceci est étendu dans [8] et [10,11] au cas de systèmes, toujours dans le cas de la méthode de Schwarz à deux niveaux. Dans ces deux travaux les problèmes aux valeurs propres qui sont résolus sont similaires car ils résultent de la même stratégie. Il s'agit d'écrire la théorie de la méthode de Schwarz à deux niveaux [6] et de résoudre des problèmes aux valeurs propres qui identifient les bons modes qui satisfont les estimation et les mauvais qui ne les satisfont pas. Les mauvais modes servent à engendrer l'espace grossier.

Nous proposons ici de généraliser cette stratégie à la méthode FETI introduite dans [1]. Le point de départ de FETI est le système de point selle (1) qui correspond au problème où les inconnues d'interfaces sont dupliquées autant de fois que le nombre de sous domaines auxquels elles appartiennent et les inconnues internes sont éliminées. La condition $B u=0$ assure que les inconnues dupliquées ont en fait la même valeur dans chacun des sous domaines. Après manipulation des équations on ramène (1) au problème (6) qui est projeté dans l'espace $V_{N}$ des incréments admissibles (4) et preconditionné par $M^{-1}$ (5). L'analyse théorique $[4,3,6]$ de ce problème montre que les valeurs propres de l'opérateur préconditionné sont toutes supérieures à 1 . Pour obtenir une borne supérieure il faut par contre ajouter des hypothèses sur les coefficients et les sous domaines, c'est cette borne que nous nous proposons de satisfaire grâce aux problèmes aux valeurs propres généralisés.

L'espace grossier ainsi que l'opérateur préconditionné correspondant sont introduits dans la Définition 3.1. Le lemme 3.2 donne l'inégalité qui est assurée grâce au choix de l'espace grossier et qui implique l'estimation sur le conditionnement de la matrice donnée dans le Théorème 3.3. Cette estimation est explicite et ne dépend que du choix d'un paramètre qui intervient dans la construction de la grille grossière et du nombre maximal de voisins que possède un sous domaine.

\section{Introduction}

It is quite intuitive that adding vectors to a coarse space can greatly improve the rate of convergence of the corresponding two level method since if the coarse space is the whole space then the method is direct. The tricky part is identifying which vectors should be used to build the coarse space in order for a good compromise between a reasonably sized coarse space and a fast converging method to be met. The idea to use eigenvalue problems to build the coarse space goes back as far as [7]. The method presented there has a convergence rate chosen a priori. In practice, the size of the coarse space may grow to be very large. In more recent work, for the case of the Darcy equation, [2] proposes, successfully, to solve a generalized eigenvalue problem to select vectors for the coarse space. This allows to reduce the size of the coarse space greatly. The idea of using generalized eigenvalue problem was used again in [8] and $[10,11]$ this time for systems of several discretized partial differential equations with, possibly, several varying coefficients. The generalized eigenvalue problems in both these approaches are very similar as they are derived using the same strategy. This strategy is to write the proof of convergence for the two 
level Schwarz preconditioner in the abstract Schwarz framework [6] until assumptions on the partition into subdomains or the coefficient distribution are needed to write an estimate (in this case the stable splitting property). Then the generalized eigenvalue problem identifies which modes don't satisfy the required estimate and these modes are dealt with by a direct solve in the coarse space. Whatever remains in the local subspaces satisfies the estimate.

Our purpose here is to generalize this strategy to the FETI (Finite Element Tearing and Interconnecting) domain decomposition method. This method was introduced by [1] and the first proof of convergence goes back to [4]. This proof is generalized by [3]. The first coarse space for FETI was introduced in [9].

The remainder of this article is organized as follows: in Section 2 we introduce the usual FETI method. Then in Section 3 we introduce the coarse space and the deflated preconditioner. Theorem 3.3 is the convergence result. Finally in Section 4 we give a few numerical results which confirm this result.

\section{FETI}

For a more complete introduction of the FETI method we refer the reader to [3]. For a given domain $\Omega \in \mathbb{R}^{d}$, a symmetric, positive definite matrix $\hat{K}$ and a right hand side $\hat{g} \in \mathbb{R}^{n}$ the original problem writes: find $\hat{u} \in \mathbb{R}^{n}$ such that $\hat{K} \hat{u}=\hat{g}$.

Suppose that the domain $\Omega$ has been partitioned into a set of $N$ non-overlapping subdomains $\Omega_{1}, \ldots, \Omega_{N}$ and that $W_{i}$ is the space of degrees of freedom on the boundary of $\Omega_{i}$. Then let $K_{i}$ be the matrix of the problem restricted to subdomain $\Omega_{i}$ and $S_{i}$ be the matrix of the same problem condensed onto the degrees of freedom in $W_{i}$ (using a Schur complement procedure). Also denote by $f_{i}$ the right hand side of the problem restricted to subdomain $\Omega_{i}$ and then condensed onto the interface (using again a Schur complement procedure). Finally, define the product space $W$, the Schur complement on this product space and the corresponding right hand side by

$$
W:=W_{1} \times \ldots W_{N}, \quad S: W \rightarrow W ; \quad S:=\left(\begin{array}{cccc}
S_{1} & 0 & \ldots & 0 \\
0 & S_{2} & \ldots & 0 \\
\ldots & \ldots & \ldots & \ldots \\
0 & 0 & \ldots & S_{N}
\end{array}\right) \text { and } f:=\left(\begin{array}{c}
f_{1} \\
\ldots \\
f_{N}
\end{array}\right) .
$$

Then the original problem rewrites in saddle point formulation: find $(u, \lambda) \in W \times U$ such that

$$
\left\{\begin{array}{l}
S u+B^{\top} \lambda=f, \\
B u=0
\end{array}\right.
$$

where $B=\left(B_{1}, \ldots, B_{N}\right)$ is a matrix which is used to ensure that the components $u_{i}$ of $u$ match at the interfaces, $\lambda$ is the vector of Lagrange multipliers used to ensure this constraint and $U:=\operatorname{range}(B)$. Given a basis for $\operatorname{Ker}(S)$ which consists of $n_{K}$ vectors, an important role will be played by the prolongation operator $R_{N}^{\top}: \mathbb{R}^{n_{K}} \rightarrow W$ which columns are these basis functions. We have used the subscript $N$ because $\operatorname{Ker}(S)$ is often referred to as the Natural coarse space for FETI. Going back to the system and using ${ }^{\dagger}$ to denote a pseudo inverse of a matrix, the first equation in (1) rewrites

$$
u=S^{\dagger}\left(f-B^{\top} \lambda\right)+R_{N}^{\top} \alpha, \text { for some } \alpha \in \mathbb{R}^{n_{K}} ; \text { as long as }\left(f-B^{\top} \lambda\right) \perp \operatorname{Ker}(S) .
$$

The additional condition ensures that the term with the pseudo inverse is well defined. It rewrites

$$
R_{N}\left(f-B^{\top} \lambda\right)=0 \Leftrightarrow G_{N}^{\top} \lambda=R_{N} f ; \text { with } G_{N}:=B R_{N}^{\top} .
$$


Injecting (2) into the second equation in (1) another saddle point formulation of the problem is: Find $(\lambda, \alpha) \in U \times \mathbb{R}^{n_{K}}$ such that

$$
\left\{\begin{array}{l}
F \lambda-G_{N} \alpha=d \\
G_{N}^{\top} \lambda=e
\end{array}, \text { where } F:=B S^{\dagger} B^{\top}, d:=B S^{\dagger} f, e:=R_{N} f, \text { and again } G_{N}=B R_{N}^{\top} .\right.
$$

In order to bring the problem down to a single equation we decompose $\lambda$ into $\lambda=\tilde{\lambda}+\lambda_{N}$ where $G_{N}^{\top} \tilde{\lambda}=0$ and $G_{N}^{\top} \lambda_{N}=e$. The choice $\lambda_{N}:=Q G_{N}\left(G_{N}^{\top} Q G_{N}\right)^{-1} R_{N} f$ fulfills the condition $G_{N}^{\top} \lambda_{N}=e$ and the problem is then: find $\tilde{\lambda} \in \operatorname{Ker}\left(G_{N}^{\top}\right)$ and $\alpha \in \mathbb{R}^{n_{K}}$ such that

$$
F \tilde{\lambda}-G_{N} \alpha=d-F \lambda_{N} .
$$

The solver for FETI is a projected conjugate gradient algorithm where the initial guess is $\lambda_{N}$ and the CG iterations take place in $\operatorname{Ker}\left(G_{N}^{\top}\right)$ to find $\tilde{\lambda}$. This is done by means of a projection operator $P_{N}$ as follows. Let $Q: U \rightarrow U$ be a self-adjoint matrix which is also positive definite on $\operatorname{range}\left(G_{N}\right)$, then define

$$
P_{N}: U \rightarrow U ; \quad P_{N}:=I-Q G_{N}\left(G_{N}^{\top} Q G_{N}\right)^{-1} G_{N}^{\top}, \text { and } V_{N}:=\operatorname{range}\left(P_{N}\right)\left(=\operatorname{Ker}\left(G_{N}^{\top}\right)\right) .
$$

Testing (3) against elements in $V_{N}$ yields the final form of the projected problem before preconditioning: find $\tilde{\lambda} \in V_{N}$ such that $P_{N}^{\top} F \tilde{\lambda}=P_{N}^{\top}\left(d-F \lambda_{N}\right)$. The so called Dirichlet preconditioner for FETI depends on the choice of a diagonal scaling matrix $D: W \rightarrow W$. It writes

$$
M^{-1}=\left[D^{-1} B^{\top}\left(B D^{-1} B^{\top}\right)^{\dagger}\right]^{\top} S\left[D^{-1} B^{\top}\left(B D^{-1} B^{\top}\right)^{\dagger}\right] .
$$

Because we solve the system using a projected conjugate gradient method we require that the search directions remain in $V_{N}$. Therefore we actually solve: find $\tilde{\lambda} \in V_{N}$ such that

$$
P_{N} M^{-1} P_{N}^{\top} F \tilde{\lambda}=P_{N} M^{-1} P_{N}^{\top}\left(d-F \lambda_{N}\right) .
$$

Lemma 2.1 Preconditioner $M^{-1}$ is self adjoint on $U$ and positive definite on range $\left(G_{N}\right)$. Consequently it is a possible choices for matrix $Q$ in the definition (4) of the natural projection operator $P_{N}$.

We are now ready to introduce the coarse space based on generalized eigenvalue problems.

\section{The coarse space}

We use again the name GenEO (which was coined in [10,11]) for Generalized Eigenproblems in the $O$ verlaps: in some sense the interfaces can be viewed as overlaps. Indeed, a degree of freedom in $W$ corresponds to a degree of freedom $k$ in the original formulation which has been duplicated as many times as the number of subdomains to which it belongs.

Definition 3.1 (GenEO coarse spaces for FETI) For each subdomain $i=1, \ldots, N$, find the eigenpairs $\left(q_{i}^{k}, \Lambda_{i}^{k}\right) \in W_{i} \times \mathbb{R}^{+}$of the generalized eigenvalue problem:

$$
S_{i} q_{i}^{k}=\Lambda_{i}^{k}\left(B_{i}^{\top} M^{-1} B_{i}\right) q_{i}^{k} .
$$

Next, given a threshold $\mathcal{K}_{i}>0$ for each subdomain, define the coarse space as

$$
U_{0}=\operatorname{span}\left\{M^{-1} B_{i} q_{i}^{k} ; 0<\Lambda_{i}^{k}<\mathcal{K}_{i}, i=1, \ldots, N\right\} .
$$

Let the interpolation operator $G_{0}^{\top}$ be the matrix whose columns are the coarse basis functions $\left\{M^{-1} B_{i} q_{i}^{k} ; 0<\right.$ $\left.\Lambda_{i}^{k}<\mathcal{K}_{i}, i=1, \ldots, N\right\}$ and let $P_{0}$ be the $\left(P_{N}^{\top} F P_{N}\right)$-orthogonal projection operator defined by

$$
P_{0}:=I-G_{0}\left(G_{0}^{\top}\left(P_{N}^{\top} F P_{N}\right) G_{0}\right)^{\dagger} G_{0}^{\top}\left(P_{N}^{\top} F P_{N}\right) .
$$


Then the two level deflation preconditioner for $F$ is

$$
\mathcal{P}_{\text {def }}^{-1}=P_{N} P_{0} M^{-1} P_{0}^{\top} P_{N}^{\top}+P_{N} G_{0}\left(G_{0}^{\top}\left(P_{N}^{\top} F P_{N}\right) G_{0}\right)^{\dagger} G_{0}^{\top} P_{N}^{\top} .
$$

The coarse space was chosen specifically to ensure that the next lemma holds.

Lemma 3.2 (Upper bound for the eigenvalues of the preconditioned operator) The following upper bound for the eigenvalues of the preconditioned operator holds

$$
\left\langle F M^{-1} \mu, M^{-1} \mu\right\rangle \leq \mathcal{N} \max _{1 \leq i \leq N}\left(\frac{1}{\mathcal{K}_{i}}\right)\left\langle M^{-1} \mu, \mu\right\rangle \text { for any } \mu \in \operatorname{range}\left(P_{0}^{\top} P_{N}^{\top}\right),
$$

where $\mathcal{N}$ is the maximal number of neighbours of a subdomain (including itself) in the sense

$$
\mathcal{N}=\max _{1 \leq i \leq N}\left(\#\left\{j ; B_{j}^{\top} B_{i} \neq 0\right\}\right) .
$$

As is proved for instance in [3] the lower bound for the eigenvalues of the preconditioned FETI method is 1 regardless of the coarse space. This remains unchanged here and so the estimate for the upper bound of the eigenvalues of the preconditioned operator in the previous lemma directly implies a bound for the condition number.

Theorem 3.3 (Main theorem for FETI with the GenEO coarse space) The condition number for FETI solved in range $\left(P_{N}\right)$ with $Q$ in the definition of $P_{N}$ chosen as $Q=M^{-1}$ and the GenEO coarse space satisfies

$$
\kappa\left(\mathcal{P}_{\text {def }}^{-1} F\right) \leq \max \left\{1, \mathcal{N} \max _{1 \leq i \leq N}\left(\frac{1}{\mathcal{K}_{i}}\right)\right\} .
$$

This bound depends only on the chosen threshold $\mathcal{K}_{i}$ we use to select eigenvectors for the coarse space and on the maximal number $\mathcal{N}$ of neighbours of a subdomain (including itself).

\section{A few numerical results}

We test the problem for two dimensional elasticity on a unit square square discretized with a simplicial mesh consisting of $101 \times 101$ nodes and $\mathbb{P}_{1}$ finite elements. The local components of the diagonal scaling matrix $D$ in the preconditioner are chosen to be the $K$-scaling matrices $D_{i}=\operatorname{diag}\left(K_{i}\right)$. The coefficient distribution is given in Figure 4 along with two partitions of the domain into 25 subdomains. In both cases the interfaces do not match the jumps in the coefficients. The results are shown in Figure 4 where $\kappa\left(\mathcal{P}_{\text {def }}^{-1} F\right)$ is the condition number of the preconditioned operator, $n_{b a d}$ is the number of bad eigenmodes selected in (7) using the threshold $\mathcal{K}_{i}$. As is expected the condition number decreases when the threshold increases. In all cases the estimate is satisfied. We also observe that for a fixed threshold more eigenmodes are used to build the coarse space in the Metis partition case. This is in agreement with the fact that this is a harder problem.

\section{Conclusion}

Thanks to generalized eigenvalue problems on the interfaces of each subdomain we have built a coarse space for FETI. The condition number of the resulting preconditioned operator does not depend on the jumps in the coefficients or the number of subdomains. Instead it depends on a chosen threshold and on the maximal number of neighbours of a subdomain. We have a proof of this result [5] and it is confirmed by preliminary numerical tests. Using the same strategy, in [5] we have also devised coarse spaces for 


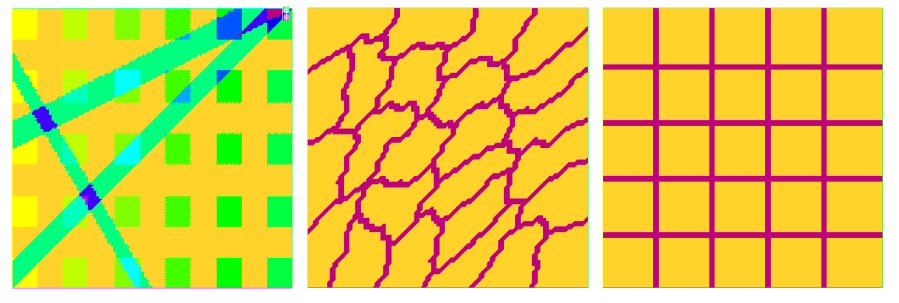

Figure 1. Left: Coefficients (Young's modulus $10^{7}<E<3 \cdot 10^{13}$; Poisson's ratio $0.3<\nu<0.4)$ - Middle: Metis partition into 25 subdomains $(1896$ interface degrees of freedom) of the unit square - Right: Regular partition into 25 subdomains (1736 interface degrees of freedom)

Gauche: Coefficients (module de Young $10^{7}<E<3 \cdot 10^{13}$; coefficient de Poisson $0.3<\nu<0.4)$ - Milieu: Décomposition Metis en 25 sous-domaines (1896 inconnues d'interface) d'un carré de taille $1 \times 1$ - Droite: Décomposition régulière en 25 sous-domaines (1736 inconnues d'interface)

\begin{tabular}{|c|c|c||c|c|}
\hline & \multicolumn{2}{|c||}{ Metis partition } & \multicolumn{2}{c|}{ Regular partition } \\
\hline $\mathcal{K}_{i}$ & $\kappa\left(\mathcal{P}_{\text {def }}^{-1} F\right)$ & $n_{\text {bad }}$ & $\kappa\left(\mathcal{P}_{\text {def }}^{-1} F\right)$ & $n_{\text {bad }}$ \\
\hline 0 & $2.9 \cdot 10^{6}$ & 0 & $1.4 \cdot 10^{5}$ & 0 \\
\hline 0.05 & 18.59 & 114 & 12.61 & 14 \\
\hline 0.1 & 10.36 & 122 & 9.01 & 19 \\
\hline 0.5 & 2.50 & 225 & 2.93 & 95 \\
\hline 1 & 1.56 & 509 & 1.32 & 238 \\
\hline 4 & 1.87 & 3295 & 1.00 & 3101 \\
\hline
\end{tabular}

Figure 2. $\kappa\left(\mathcal{P}_{\text {def }}^{-1} F\right)$ and $n_{\text {bad }}$ vs. $\mathcal{K}_{i}$ $\kappa\left(\mathcal{P}_{\text {def }}^{-1} F\right)$ et $n_{\text {bad }}$ contre $\mathcal{K}_{i}$

FETI with the, cheaper, lumped preconditioner and also for BDD (Balancing Domain Decomposition). There we prove that the same convergence result as the one in Theorem 3.3 holds in both these cases.

\section{References}

[1] C. Farhat, F.-X. Roux, A method of finite element tearing and interconnecting and its parallel solution algorithm, International Journal for Numerical Methods in Engineering, 32:12051227, 1991.

[2] J. Galvis and Y. Efendiev, Domain decomposition preconditioners for multiscale flows in high contrast media: Reduced dimension coarse spaces, Multiscale Modeling and Simulation, 8(5):16211644, 2010.

[3] A. Klawonn, O.B. Widlund, FETI and Neumann-Neumann iterative substructuring methods: connections and new results, Communications on pure and applied Mathematics, 54(1):5790, 2001.

[4] J. Mandel, R. Tezaur, Convergence of a substructuring method with Lagrange multipliers, Numerische Mathematik, 73(4):473487, 1996.

[5] N. Spillane, D. Rixen Spectral coarse spaces for robust FETI and BDD algorithms, in preparation, 2012.

[6] A. Toselli, O. Widlund Domain decomposition methods: algorithms and theory, Springer, 2005.

[7] M. Brezina, C. Heberton, J. Mandel, P. VAnĚK, An iterative method with convergence rate chosen a priori Technical Report 140, University of Colorado Denver, CCM, University of Colorado Denver, April 1999.

[8] Y. Efendiev, J. Galvis and R. Lazarov, J. Willems, Robust domain decomposition preconditioners for abstract symmetric positive definite bilinear forms, ESAIM: Mathematical Modelling and Numerical Analysis, 46(05):11751199, 2012 .

[9] C. Farhat, P.S. Chen, J. Mandel, A scalable Lagrange multiplier based domain decomposition method for timedependent problems, International Journal for Numerical Methods in Engineering, 38(22):38313853, 1995.

[10] N. Spillane, V. Dolean, P. Hauret, F. Nataf, C. Pechstein, R. Scheichl, A Robust Two Level Domain Decomposition Preconditioner for Systems of PDEs, Comptes Rendus Mathématique, 349(23-24):12551259, 2011.

[11] N. Spillane, V. Dolean, P. Hauret, F. Nataf, C. Pechstein, R. Scheichl, Abstract Robust Coarse Spaces for Systems of PDEs via Generalized Eigenproblems in the Overlaps, NuMa-Report, 2011-07, 2007. 